\title{
Introduction: The Evolution of Chaos
}

I am an old man now, and when I die and go to Heaven there are two matters on which $\mathrm{I}$ hope for enlightenment. One is quantum electrodynamics, and the other is the turbulent motion of fluids. And about the former I am really rather optimistic.

- Sir Horace LAMB, 1932

I $T$ all started with the moon. If only the earth could have gone 1 round the sun by itself, unperturbed by the complications in its orbit which the moon's gravitational field introduced, Newton's equations of motion would have worked fine. But when the moon entered the picture, the situation became too complex for simple dynamics to handle. The moon attracted the earth, causing perturbations in the earth's orbit which changed the earth's distance from the sun, which in turn altered the moon's orbit around the earth, which meant that the original basis for the calculations had changed and one had to start over from the beginning. The problem was sufficiently complex and interesting to merit a name and a prize of its own. It became known as the three-body problem, and the king of Sweden offered a reward to the first person who could prove a solution was possible.' Instead, in I 890 Henri Poincaré (whose formidable talent was responsible for creating topology and half a dozen other new fields) published a paper proving that in general, a solution was not possible by means of Newtonian equations (Poincaré,

'I am indebted to Phillip Holmes for drawing this incident to my attention. 
I 890). With commendable foresight, the king of Sweden gave Poincaré the prize anyway. Perhaps he intuited that Poincarés work had opened a window on a kind of world that Newtonian mechanics had not envisioned. By proving that the introduction of small perturbations into linear equations was not in general sufficient to solve nonlinear problems, Poincaré implied that a new kind of science and mathematics was necessary to account for the dynamics of complex systems. From this realization the science of chaos was born.

But not immediately. Positivism was in full swing throughout Western Europe and America, and mathematicians were preoccupied by efforts to put mathematics on a firm foundation by formalizing it. By I93I, when Kurt Gödel dashed these hopes by proving that formal systems could not be axiomatized completely (Gödel, I962), the clue that Poincaré's work provided for the labyrinthine difficulties of complex dynamics was in danger of dropping out of sight. (An exception was Russia, where many important results were obtained during the I930s and I940s.) In the West, the study of complex dynamics did not come into its own until computers became widespread and readily accessible during the I960s and I970s.

The same two decades saw a significant intellectual shift throughout the human sciences. Its essence was a break away from universalizing, totalizing perspectives and a move toward local, fractured systems and modes of analysis. Just as new methods were being developed within the physical sciences to cope with the complexities of nonlinear systems, so new ways of reading and writing about literature were coming to the fore in critical theory. The (old) New Critics had taken for granted that a literary work was a verbal object, bounded and finite, however ambiguous it might be within. But the (new) New Critics saw textual boundaries as arbitrary constructions whose configurations depended on who was reading, and why. As books became texts, they were transformed from ordered sets of words to permeable membranes through which flowed the currents of history, language, and culture. Always already lacking a ground for their systems of signification, texts were not deterministic or predictable. Instead they were capable of becoming unstable whenever the slightest perturbation was introduced. The well-wrought urn, it seemed, was actually a reservoir of chaos. 
Each of these developments appeared within a well-defined disciplinary tradition, and each is explicable in terms of what had preceded it within the discipline. Nonlinear dynamics, for example, traces its linage through Mitchell Feigenbaum and Edward Lorenz back to Poincaré; poststructuralism through such theorists as Jacques Derrida and Paul de Man back to Friedrich Nietzsche and Martin Heidegger. From the specialist's point of view, there is no need to go outside these boundaries to understand what happened. But there are also suggestive similarities across disciplinary lines. Suppose an island breaks through the surface of the water, then another and another, until the sea is dotted with islands. Each has its own ecology, terrain, and morphology. One can recognize these distinctions and at the same time wonder whether they are all part of an emerging mountain range, connected both through substrata they share and through the larger forces that brought them into being.

In this book, I argue that certain areas within the culture form what might be called an archipelago of chaos. The connecting theme is a shift in the way chaos is seen; the crucial turn comes when chaos is envisioned not as an absence or void but as a positive force in its own right. This is a three-sided study, triangulating among chaos theory, poststructuralism, and contemporary fiction. Also of concern is the cultural matrix from which all three sides emerge and with which they interact. Concerned with the physical sciences as well as literature, the study investigates language's power to constitute reality, and reality's power to constrain and direct language. It speculates about the broader cultural conditions that authorize the new visions of chaos, and inquires into how these conditions shape and are shaped by modern narratives.

The metaphor of the triangle implies, of course, that there are connections and relationships among the three sides. One of the challenges in literature and science is to develop methodologies that can illuminate convergences between disciplines, while still acknowledging the very real differences that exist. In my view, analogies between literary and scientific versions of chaos are important both for the similarities they suggest and for the dissimilarities they reveal. The similarities arose because of broadly based movements within the culture which made the deep assumptions underlying the new paradigms thinkable, perhaps inevitable, thoughts. They illustrate 
how feedback loops among theory, technology, and culture develop and expand into complex connections between literature and science which are mediated through the cultural matrix. The dissimilarities, by contrast, point to the importance of disciplinary traditions in guiding inquiry and shaping thought. To account for them, it is necessary to understand how and why certain questions became important in various disciplines before the appearance of the new paradigms. The dual emphasis on cultural fields and disciplinary sites implies a universe of discourse that is at once fragmented and unified. Cultural fields bespeak the interconnectedness of a world in which instantaneous global communication is a mundane reality; local differences acknowledge the power of specialization within contemporary organizations of knowledge.

The connections I explore among contemporary literature, critical theory, and science are not generally explainable by direct influence. Rather, they derive from the fact that writers, critics, and scientists, however specialized or esoteric their work, all share certain kinds of everyday experiences. Consider the following question: Why should John Cage become interested in experimenting with stochastic variations in music about the same time that Roland Barthes was extolling the virtues of noisy interpretations of literature and Edward Lorenz was noticing the effect of small uncertainties on the nonlinear equations that described weather formations? An influence argument would look for direct connections to explain these convergences. Sometimes such connections exist. It is possible that Barthes listened to Cage, Cage studied Lorenz, Lorenz read Barthes. But it stands to reason that, of all the interdisciplinary parallels one might notice, only a few will be connected by direct lines of influence, which are usually conveyed through disciplinary traditions. One could, for example, trace a clear line of descent from John Cage to Brian Eno to the Talking Heads and U2.

Interdisciplinary parallels commonly operate according to a different dynamic. Here influence spreads out through a diffuse network of everyday experiences that range from reading The New York Times to using bank cards on automatic teller machines to watching MTV. When enough of the implications in these activities point in the same direction, they create a cultural field within which certain questions or concepts become highly charged. Perhaps, for example, 
Brian Eno might first learn about Roland Barthes through Time magazine. Intrigued, he might read one of Barthes's books. Or he might not. The brief article summarizing Barthes's ideas would then become one of the elements in Eno's cultural field, available to be reinforced by other elements until a resonance built up which was strong enough to be a contributing factor in his work.

Between 1960 and 1980 , cultural fields were configured so as to energize questions about how stochastic variations in complex systems affected systemic evolution and stability. It is easy to see how the political movements of the I960s contributed to this interest. Also important was the growing realization that the world itself had become (or already was) a complex system economically, technologically, environmentally. Along with the information capabilities of modern communication systems came the awareness that small fluctuations on the microscale could, under appropriate conditions, quickly propagate through the system, resulting in large-scale instabilities or reorganizations. A revolution in the Middle East, for example, could trigger a precipitous rise in oil prices, leading to energy shortages and inflationary spirals in the developed countries, which in turn could spark a global recession that would force major restructurings in international finance. When such cascading scenarios are ever-present possibilities, the realization that small causes can lead to very large effects is never far from consciousness. The ecological movement is a case in point. People concerned about the global environment are intensely aware that a seemingly small event-an inattentive helmsman on the bridge of an oil tanker, say-can have immediate and large-scale effects on an entire coastal area. Implicit in this awareness is increased attention to random fluctuations, and consequently to the role that chaos plays in the evolution of complex systems.

Another factor that helped to energize the concepts underlying the new paradigms was the realization that as systems became more complex and encompassing, they could also become more oppressive. In more than one sense, the Cold War brought totalitarianism home to Americans. As information networks expanded and data banks interlocked with one another, the new technology promised a level of control never before possible. In this paranoiac atmosphere, chaotic fluctuations take on an ambiguous value. From one point of 
view they threaten the stability of the system. From another, they offer the liberating possibility that one may escape the informational net by slipping along its interstices. In Gravity's Rainbow, for example, chaos reigns supreme in the "Zone," the free-floating, anarchical space that was Western Europe for a brief time at the end of World War II. Threatening as the Zone sometimes is, its chaotic multivalency marks the distance between Pynchon's postmodern text and the nightmare vision of Orwell's 1984 .

In the assigning of a positive value to chaos, information theories and technologies played central roles. In addition to creating the necessary technological landscape, they laid the theoretical foundation for conceptualizing chaos as a presence rather than an absence. Later chapters will explore this transformation, showing how a crucial move in the transvaluation of chaos was the separation of information from meaning. Once this distinction was made, the way was open for information to be defined as a mathematical function that depended solely on the distribution of message elements, independent of whether the message had any meaning for a receiver. And this step in turn made it possible to see chaotic systems as rich in information rather than poor in order.

Suppose I send a message that contains the series $2,4,6,8 \ldots$ and ask you to continue the sequence. Because you grasp the underlying pattern, you can expand the series indefinitely even though only a few numbers are specified. Information that has a pattern can be compressed into more compact form. I could have sent the message as "Enumerate the even integers, starting with 2." Or even more concisely, "Count by twos." By contrast, suppose I send you the output of a random number generator. No matter how many numbers I transmit, you will be unable to continue the sequence on your own. Every number comes as a surprise; every number conveys new information. By this reasoning, the more random or chaotic a message is, the more information it contains.

You may object that although the numbers are always new and surprising, they do not mean anything. The objection illustrates why it was necessary to separate information from meaning if chaotic systems were to be considered rich in information. Implicit in the transvaluation of chaos is the assumption that the production of information is good in itself, independent of what it means. Having 
opened this possibility by creating a formal theoretical framework that implied it, information and communication technologies actualized it in everyday life. Every time we keep a TV or radio going in the background, even though we are not really listening to it, we are acting out a behavior that helps to reinforce and deepen the attitudes that underwrite a positive view of chaos.

Stanislaw Lem in The Cyberiad has a fable that speaks to this point (Lem, r 974b). Two constructors, Trurl and Klapaucius, take a journey that brings them into the clutches of Pugg the PHT Pirate. Actually the name (which they know only from rumor) is a slight error. Pugg has a Ph.D, and what he craves above all else, even more than gold and obeisance from his subjects, is information. So Trurl and Klapaucius create for him a Demon of the Second Kind.

The First Kind of Demon (about which we will hear more later) was proposed by James Clerk Maxwell in 1859 . To test the second law of thermodynamics, Maxwell imagined a mythical imp who presided over a box of ideal gas divided by a partition. The Demon's task was to sort the molecules by opening and closing a shutter in the partition, allowing only the fast molecules to pass through. The resulting separation created a temperature differential, which in turn could be converted into work. Lem refers to this history by allusion to its difference from the Demon of the Second Kind, who is an upto-date version appropriate to an information age. Like his predecessor, the Second Demon also presides over a box of stale air. Instead of sorting the molecules, however, he watches their endless dance. Whenever the molecules form words that make sense, he writes them down with a tiny diamond-tipped pen on a paper tape. Whereas the First Demon uses randomness to produce work, the Second Demon uses it to produce information.

Pugg is delighted with the invention and immediately sits down to read the tape with his hundreds of eyes. He learns "how exactly Harlebardonian wrigglers wriggle, and that the daughter of King Petrolius of Labondia is named Humpinella, and what Frederick the Second, one of the paleface kings, had for lunch before he declared war against the Gwendoliths, and how many electron shells an atom of thermionolium would have, if such an element existed, and what is the cloacal diameter of a small bird called the tufted twit" ( $p$. r 57). As the list continues and the tape rolls on, Pugg is buried un- 
der its toils. The narrator informs us that he sits there to this day, learning "no end of things about rickshaws, rents and roaches, and about his own fate, which has been related here, for that too is included in some section of the tape-as are the histories, accounts and prophecies of all things in creation, up until the day the sun burns out; and there is no hope for him ... unless of course the tape runs out, for lack of paper" (pp. I 59-160).

The fable is at least as compelling today as it was when it was written in 1967 . Like Pugg, we are increasingly aware that information is a commodity every bit as valuable as diamonds and gold. Indeed, it can often be converted directly into money (as recent insider trading scandals have demonstrated). What are the computer programs that large investment firms use for stock trading but Demons of the Second Kind? From random fluctuations in the market they extract information and money, thus justifying Maxwell's intuition that the second law of thermodynamics may have left something important out of account. Whether this project will succeed in the long run or bury us underneath it, as Pugg was entombed by his information, remains to be seen. Not in doubt is the important role that such phenomena play in reinforcing the connection between information and randomness. The more chaotic a system is, the more information it produces. This perception is at the heart of the transvaluation of chaos, for it enables chaos to be conceived as an inexhaustible ocean of information rather than as a void signifying absence.

Once the link was forged between chaos and information, a chain of consequences followed. To introduce them, I want to explain more about the structure and content of chaos theory. First, a disclaimer: "chaos theory" and the "science of chaos" are not phrases usually employed by researchers who work in these fields. They prefer to designate their area as nonlinear dynamics, dynamical systems theory, or, more modestly yet, dynamical systems methods. To them, using "chaos theory" or the "science of chaos" signals that one is a dilettante rather than an expert. Nevertheless, I will use these terms throughout my discussion, because part of my project is to explore what happens when a word such as "chaos," invested with a rich tradition of mythic and literary significance, is appropriated by the sciences and given a more specialized meaning. The older 
resonances do not disappear. They linger on, creating an aura of mystery and excitement that even the more conservative investigators into dynamical systems methods find hard to resist (especially when they apply for grants or explain their work to the public). As new meanings compete with traditional understandings within the sign of chaos, "chaos" becomes a highly charged signifier, attracting interest from many areas within the culture. The underlying forces that have fueled the new paradigms-the rapid development of information technologies, the increasing awareness of global complexities, and consequent attention to small fluctuations-do not depend on any single factor, especially one so slight as the choice of a name for the new theories. But the name is important, for in its multiple meanings it serves as a crossroads at which diverse paths within the culture meet.

Chaos theory is a wide-ranging interdisciplinary research front that includes work in such fields as nonlinear dynamics, irreversible thermodynamics, meteorology, and epidemiology. It can be generally understood as the study of complex systems, in which the nonlinear problems that perplexed Poincaré's contemporaries are considered in their own right, rather than as inconvenient deviations from linearity. Within chaos theory, two general emphases exist. In the first, chaos is seen as order's precursor and partner, rather than as its opposite. The focus here is on the spontaneous emergence of self-organization from chaos; or, in the parlance of the field, on the dissipative structures that arise in systems far from equilibrium, where entropy production is high. The realization that entropy-rich systems facilitate rather than impede self-organization was an important turning point in the contemporary reevaluation of chaos. A central figure in this research is Ilya Prigogine, who in I 977 won the Nobel Prize for his work with irreversible thermodynamics. The title of the book he co-authored with Isabelle Stengers, Order out of Chaos, provides the motto for this branch of chaos theory.

The second branch emphasizes the hidden order that exists within chaotic systems. Chaos in this usage is distinct from true randomness, because it can be shown to contain deeply encoded structures called "strange attractors." Whereas truly random systems show no discernible pattern when they are mapped into phase space, chaotic systems contract to a confined region and trace complex patterns 
within it. The discovery that chaos possesses deep structures of order is all the more remarkable because of the wide range of systems that demonstrate this behavior. They range from lynx fur returns to outbreaks of measles epidemics, from the rise and fall of the Nile River to eye movements in schizophrenics. Researchers associated with this branch of chaos theory include Edward Lorenz, Mitchell Feigenbaum, Benoit Mandelbrot, and Robert Shaw. The strange-attractor branch differs from the order-out-of-chaos paradigm in its attention to systems that remain chaotic. For them the focus is on the orderly descent into chaos rather than on the organized structures that emerge from chaos.

For a variety of reasons, fewer connections have been forged between the two branches than one might expect. The two branches employ different mathematical techniques to analyze chaos. Although some translations have been made, the different modes of analysis make communication between the branches difficult. There are also different views on what the research signifies. Prigogine has strong ties with French intellectual circles, and the order-out-ofchaos branch is known for its willingness to extrapolate beyond experimental results to philosophical implications. It has been criticized within the scientific community for the relative paucity of its results, especially in light of the large philosophical claims made for them. The strange-attractor branch, by contrast, has been if anything undertheorized; its practioners prefer to concentrate on problems of immediate practical interest. In brief, the order-out-of-chaos branch has more philosophy than results, the strange-attractor branch more results than philosophy.

These different orientations lead to different kinds of conclusions. Prigogine sees the primary importance of the order-out-of-chaos branch in its ability to resolve a long-standing metaphysical problem: it reconciles being with becoming. For him, chaos theory is revolutionary because of what it can tell us about the arrow of time. By comparison, the strange-attractor branch emphasizes the ability of chaotic systems to generate new information. Almost but not quite repeating themselves, chaotic systems generate patterns of extreme complexity, in which areas of symmetry are intermixed with asymmetry down through all scales of magnification. For researchers in this branch, the important conclusion is that nature, too complex 
to fit into the Procrustean bed of linear dynamics, can renew itself precisely because it is rich in disorder and surprise.

Perhaps because of these differences, James Gleick, in his influential narrative history of chaos theory (Chaos: Making a New Science, 1987), does not acknowledge that more than one branch exists. He barely mentions Prigogine's name in passing, describing his work as springing from a "highly individual, philosophical view" ( $\mathrm{p}$. 339). This remarkable omission testifies to how contested the name of chaos is, even within the physical sciences. (Some researchers in dynamical systems theory think that Gleick went too far in calling it a new science.) Nevertheless, there are points of convergence between the two branches. For example, the Belousov-Zhabotinskii reaction, which serves as a prime example of a self-organizing system, has also been shown to contain a strange attractor. In the face of these commonalities, that so definite a breach should exist has interesting political as well as philosophical dimensions, some of which will be touched upon in chapter 4 , where Prigogine's work is discussed.

Despite the breach, it is possible to identify several characteristics that chaotic systems share. These characteristics will be discussed in detail in later chapters; it may be useful to indicate briefly here what they are. Perhaps the most general is nonlinearity. With linear equations, the magnitudes of cause and effect generally correspond. Small causes give rise to small effects, large causes to large effects. Linearity connotes this kind of proportionality. Equations that demonstrate it can be mapped as straight lines or planes.

Nonlinear functions, by contrast, connote an often startling incongruity between cause and effect, so that a small cause can give rise to a large effect. There is a good reason why linear equations have dominated the study of dynamical systems: nonlinear differential equations do not generally have explicit solutions. If nonlinear equations are introduced at all into physics courses, they are frequently relegated to the final hectic week or two of the course. The practice reinforces the assumption, implicit in Newtonian mechanics and encoded within the linguistic structure of stem and prefix, that linearity is the rule of nature, nonlinearity the exception. Chaos theory has revealed that in fact the opposite is true. To illustrate the predominance of nonlinear systems, Gleick quotes the mathematician 
Stanislaw Ulam's quip that calling the science of chaos the study of nonlinear systems is like calling zoology the "study of nonelephant animals" (p. 68).

Take the weather. Even with large computers, it is impossible to predict weather patterns accurately more than a couple of days in advance. Weather prediction is difficult because small fluctuations quickly amplify into large-scale changes. Gleick estimates that if sensing devices were placed at one-foot intervals on poles as high as the atmosphere, and these poles were placed one foot apart all over the earth and the results fed into a supercomputer, the weather could still not be accurately predicted. In between the sensing devices, fluctuations in temperature and wind velocity would go unrecorded, and these fluctuations could soon affect global weather patterns (Gleick, I987:2I). The ability of minute fluctuations to cause large-scale changes holds for a wide variety of systems, from cream swirling in coffee to the thundering turbulence of Niagara Falls. Chaos is all around us, even in the swinging pendulum that for the eighteenth century was emblematic of a clockwork universe.'

Another characteristic of chaotic systems is contributed by their complex forms, which lead to a new awareness of the importance of scale. The new models bring into question an assumption so deeply woven into classical paradigms that it is difficult to see that it is an assumption. In classical physics, objects are considered to be independent of the scale chosen to measure them. A circle is assumed to have a set circumference, whether it is measured with a yardstick or with a ruler an inch long. Classical paradigms grant that smaller rulers may yield more precision than larger ones. But these differences are considered to be merely empirical variations that do not affect the existence of a "true" answer. This assumption works well for regular forms, such as circles, rectangles, and triangles. It does not work well for complex irregular forms-coastlines, for example, or mountain landscapes, or the complex branchings of the human vascular system. Here measurements on scales of different lengths do not converge to a limit but continue to increase as measurement scales decrease. Fractal geometry (a neologism coined by Benoit Mandelbrot, its inventor) expresses this complexity through in-

'For a discussion of chaotic pendulum swings, see Tritton, I 986. 
creased dimensionality. Fractal forms possess additional fractions of dimensions, with the fractionality corresponding to the degree of roughness or irregularity in the figure.

Nonlinear dynamics, another important area within the sciences of chaos, is akin to fractal geometry in that it posits a qualitative and not merely a quantitative difference between linear and complex systems. Turbulent flow, for example, possesses so many coupled degrees of freedom that even the new supercomputers are inadequate to handle the required calculations. Since doing more of the same kind of calculations that one would use for laminar flow does not usually yield a solution, the difference between turbulent and laminar flow amounts to a qualitative distinction, an indication that another kind of approach is needed.

An essential component of this approach is a shift in focus from the individual unit to recursive symmetries between scale levels. For example, turbulent flow can be modeled as small swirls within larger swirls, nested in turn within still larger swirls. Rather than trying to follow an individual molecule, as one might for laminar flows, this approach models turbulence through symmetries that are replicated over many scale levels. The different levels are considered to be connected through coupling points. At any one of these coupling points, minute fluctuations can cause the flow to evolve differently, so that it is impossible to predict how the system will behave. The authors of a recent article on chaos in Scientific American illustrate the point by estimating that if an effect as small as the gravitational pull of an electron at the edge of the galaxy is neglected, the trajectories of colliding billiard balls become unpredictable within one minute. ${ }^{3}$

The comparison of chaos theory with quantum mechanics is illuminating in this respect. As is well known, quantum mechanics has important implications for the precision with which subatomic reality can be known. However, these implications are largely irrelevant for macroscopic bodies that remain stationary or move at ordinary speeds. The uncertainty principle does not alter the fact that tables and chairs stay neatly in place unless they are moved. Chaos theory, by contrast, studies systems configured so as to bring small uncertainties quickly up to macroscopic expression. Even a microscopic

Crutchfield, Farmer, Packard, and Shaw, I 986. 
fluctuation can send a chaotic system off in a new direction. This observation leads to another important characteristic of complex systems-their sensitivity to initial conditions. Quantum mechanics dovetails with chaos theory because it guarantees that there will always be some minimal level of fluctuation. An appropriately configured system will amplify these initial uncertainties until they are evident even on the macroscale.

To see why some systems magnify uncertainties and others do not, compare pushing alphabet blocks around with trying to get a rapidly moving bowling ball to move smoothly down the lane. Small hand tremors do not affect one's ability to position the blocks precisely, for the blocks have relatively large stabilizing surfaces. The bowling ball, by contrast, touches the alley at only a small portion of its surface, and its curvature makes it extremely sensitive to small motion tremors. Bowling is a difficult sport because a ball thrown in nearly identical ways can nevertheless follow very different paths. One time it may curve just right for a strike and another time veer off into the gutter, even though it was thrown almost the same both times. This extreme sensitivity to initial conditions is characteristic of chaotic systems. In fact, unless the starting conditions can be specified with infinite precision, chaotic systems quickly become unpredictable. Chaotic systems thus combine qualities that classical science considered antithetical and that quantum mechanics does not anticipate. Chaotic systems are both deterministic and unpredictable.

Other characteristics that complex systems share are feedback mechanisms that create loops in which output feeds back into the system as input. In certain chemical reactions, for example, a product may also serve as a catalyst for the reaction, driving it to generate more product, which in turn becomes more catalyst. The resulting dynamics are instrumental in explaining why organized structures can spontaneously emerge from initially small perturbations in the solution. In computer modeling of mathematical functions, iteration operates according to a similar principle, the output of one calculation serving as input for the next. When the function is strongly nonlinear, small fluctuations in the data are not smoothed out as iteration proceeds. Rather, they are magnified through a cas- 
cading series of bifurcations. In physical systems similar mechanisms are associated with the onset of turbulence, so that microscopic fluctuations can be amplified until they affect macroscopic flow.

The orderly disorder of chaotic systems had no recognized place within classical mechanics. By demonstrating that such systems not only exist but are common, chaos theory has in effect opened up, or more precisely brought into view, a third territory that lies between order and disorder. Does this mean that classical ideas of order have been repudiated? Here I think we must be cautious in drawing inferences about what the new sciences imply for the humanities. Chaos theory has a double edge that makes appropriations of it problematic for humanistic arguments that want to oppose it to totalizing views. On the one hand, chaos theory implies that Newtonian mechanism is much more limited in its applicability than Laplace supposed. On the other hand, it aims to tame the unruliness of turbulence by bringing it within the scope of mathematical modeling and scientific theory. It promises to provide at least some of the answers that Sir Horace Lamb, quoted in the epigraph, doubted even God could provide. From this perspective, chaos theory does not undermine an omniscient view. Rather, it extends it beyond where even Newtonian mechanics could reach. In this respect it is profoundly unlike most poststructuralist literary theories, especially deconstruction.

I am consequently wary of the claim that chaos theory provides confirmation from within the physical sciences that totalizing perspectives are no longer valid. ${ }^{4}$ In my view, this inference at once overestimates and underestimates the significance of chaos theory. It is an overestimation because chaos theory is not opposed to normal science; it is normal science. It follows the same procedure as any other scientific discipline in accrediting its members, devising research protocols, and evaluating results. Like other sciences, it agrees that parsimonious explanations are better than multiplicitous ones. Mitchell Feigenbaum's name for his discovery that nonlinear

"See, for example, the discussion of "paralogy" in Lyotard, 1984. Alvin Toffler comes close to saying the same thing in his Foreword to Prigogine and Stenger, 1984 :xi-xxvi. 
dissipative systems follow a predictable path to chaos indicates how far the sciences of chaos are from the critique of universalism within the humanities: he called it universality theory. ${ }^{5}$

Nevertheless, it would be a mistake to think that chaos theory has no significant consequences for the humanities. On a deep level, it embodies assumptions that bring into question presuppositions that have underlain scientific conceptualizations for the last three hundred years. Among the challenges it poses are whether an effect is proportional to a cause; how scale variance affects facts; what it means to model a physical system; and what cosmological scenario we are taking part in. Through its concern with the conditions that make movement from local sites to global systems possible, it exposes presuppositions within older paradigms that made universalization appear axiomatic. In this sense it is an underestimation to say that chaos theory has redefined what science means. Changed are not the disciplinary procedures and criteria of normal science but the epistemic ground on which it-and much else in contemporary culture-rests.

When a dichotomy as central to Western thought as order/disorder is destabilized, it is no exaggeration to say that a major fault line has developed in the episteme. It would be strange indeed if there were not other theoretical enterprises that also work this fault line. ${ }^{6}$ Some of the most visible are within poststructuralism, especially deconstruction. Just as the new scientific paradigms challenge the primacy traditionally accorded to ordered systems, so deconstruction exposes the interrelation between traditional ideas of order and oppressive ideologies. The scientific theories show that deterministic physical systems become chaotic because initial conditions cannot be specified with infinite accuracy; deconstructive readings operate upon texts to reveal the indeterminacy that results from the lack of an absolute ground for language. The scientific paradigms embody a shift of perspective away from the individual unit to recursive sym-

'Feigenbaum, 1980. It is not a coincidence that Einstein also considered naming his theory "universal invariance" before settling on "relativity."

"Among the important challenges to totalizing views which I will not discuss are Richard Rorty's Philosophy and the Mirror of Nature (Princeton: Princeton University Press, 1979) and Consequences of Pragmatism: Essays, 1972-1980 (Minneapolis: University of Minnesota Press, 1982). 
metries; poststructuralism, especially as exemplified in the work of Michel Foucault, writes about the death of the subject and the couplings that allow changes to sweep rapidly through a culture. The science of chaos reveals a territory that cannot be assimilated into either order or disorder; deconstruction detects a trace that cannot be assimilated into the binary oppositions it deconstructs. These correspondences are not accidental. They reflect what Christine Froula (1985), in comparing deconstruction with quantum mechanics, identified as a deepening crisis of representation in Western thought.

Because I discussed the scientific models first, I may have given the impression that they stimulated the development of analogous theories in literature. This is almost certainly not the case. The literary theories appeared simultaneously with, or slightly before, cognate formations in science. Postmodernism was already well articulated within contemporary literature and critical theory by I.970 and has precursors at least as early as Nietzsche. By comparison, Lorenz's seminal paper "Deterministic Nonperiodic Flow," first published in 1963, did not attract widespread attention (as indicated by frequency of citation) until about a decade later. ${ }^{7}$ The chronology does not correspond with the tacit acceptance of scientific priority which for many years informed influence studies in literature and science (an acceptance I have challenged elsewhere as a reinscription of the scientism prevalent throughout the academy in the r93os and I940s). ${ }^{8}$ From my point of view, an additional attraction of the new paradigms is their built-in resistance to this traditional mode of explanation. Even if I did not favor a cultural explanation, I would be thrown back upon it by the facts of the case, unless I were willing to grant that the correspondences are coincidence.

I have been construing the division between literature and science as the difference between literary theory on the one hand and scientific models on the other. Now I want to cleave the copula in

Gleick points out that Lorenz's paper was not noticed for some time (I987:3 I).

${ }^{*} \mathrm{~N}$. Katherine Hayles, "Reconfiguring Literature and Science: From Supplementarity to Complementarity," in First-Born Affinities: Theories of Literature and Theories of Science, ed. Paul Privateer and George Rousseau (Carbondale: Southern Illinois University Press, forthcoming), and "Turbulence in Literature and Science: Questions of Influence," in American Literature and Science, ed. Robert Scholnick (Chapel Hill: University of North Carolina Press, forthcoming). 
another way, putting critical and scientific theory on one side and literature on the other. Whereas the earlier division facilitated comparisons between literary and scientific concepts, this construction brings into play distinctions between disciplinary and nondisciplinary work. To work within a discipline is to be trained in such a way as to absorb the practices, knowledge, and presuppositions that define the discipline. Among the practices that maintain and replicate disciplinary presuppositions are graduate advisory systems, course contents and selections, comprehensive examinations, and dissertation defenses. Different as literature and science are, they are both clearly disciplines in this sense.

Creative writers also work within traditions and read one another's work. But because they are not accredited in the same way, they have considerably more latitude in defining the contexts appropriate to evaluation of their work. Even for writers who come out of university writers' workshops (such as the one at the University of Iowa, where I teach), there is a very significant difference between the command they are expected to demonstrate over disciplinary traditions and that required from a graduate student in the English department. The accreditation most important for the creative writer cannot, in fact, be conferred by an institution. It comes largely from presses and magazines willing to buy the work, and thus from readers rather than peers and colleagues. This orientation toward readership and the relative freedom from academic specialization make creative writing more responsive to the culture in general than a strictly defined disciplinary tradition. As a result, it tends to carry with it more of the substrata of earlier cultural formations than does contemporary writing within disciplines.

I risk belaboring this rather obvious point because it has important implications for the way literature, as distinct from disciplinary writing, interacts with the new paradigms. Responding to a more diverse audience, creative writers are forced into an awareness of how stratified and heterogeneous culture is in a way that disciplinary writers are not. A physicist who works in plasma physics told me that perhaps three hundred people in the country read and understood his work, and that he personally knew 90 percent of them. Many literary critics could say the same. But what creative writer, if 
she hopes to thrive professionally, could afford to appeal to an audience this small and specialized?

Moreover, creative writing is located within complex fields of intertextual resonances that affect signification not only in the narrow sense of the way words are understood but also in the broader sense of the way plots are structured, characters conceived, actions represented. Combined, these factors make the literary texts more concerned than either chaos theory or deconstruction with the aura of cultural meanings that surrounds chaos. It is therefore important for my discussion of literature (and also for literary theory and science) to explore what chaos meant in its traditional guise as mythopoeic concept, before it acquired its contemporary connotations of maximum information, dissipative reorganization, and deeply encoded structure. These older resonances modify and partially transfigure new versions of chaos in literature, even as they are in the process of being articulated.

Within the Western tradition, chaos was associated with the unformed, the unthought, the unfilled, the unordered. Hesiod in the Theogony designates Chaos as that which existed before anything else, when the universe was in a completely undifferentiated state. Later in the Theogony, he uses the term to signify the gap that appeared when Heaven separated from Earth through the influence of Eros. Chaos and Eros thus have a mysterious connection as the two primeval forces of the world, although Chaos is the older of the two. According to Hesiod, Heaven and Earth embraced, rain fell, and the wet and dry, cold and hot became distinct from one another, thereafter combining in various proportions to form the universe. Narrating the birth of the world as a story of increasing differentiation of form, the Theogony depicts chaos both as not-form and the background against which the creation of form takes place.

The tradition that identified Chaos as that which existed when the world did not continued at least through the Renaissance. In Paradise Lost, God creates the world not out of nothing but out of Chaos, the primeval materia of the universe before it was invested with spirit. Also continuing into the Renaissance was the apposition between Eros and Chaos as the two primeval forces of the world. It occurs in Shakespeare, for example, although the tonalities are sig- 
nificantly darker than in Hesiod. In Shakespeare a return to Chaos signals a failure of love, as though the universe were disintegrating back to where it was before Eros appeared. Othello tells Desdemona, "Perdition catch my soul, / But I do love thee! and when I love thee not, / Chaos is come again" (Othello, III.iii,90-92). Similarly, when Venus mourns Adonis in Shakespeare's poem, we are told that "beauty dead, black chaos comes again" (Venus and Adonis, 1.1020). The catastrophic implications of a return to Chaos become explicit in Ulysses' great speech on degree, where "Chaos, when degree is suffocate, / Follows the choking" (Troilus and Cressida, I.iii.r 25-126). In the same vein Gloucester, soon to become Richard III, says that he is unsuited to be a "ladies man" because his misshapen body is "like to a chaos" (Henry VI, pt. 3, III.ii. I6I).

During the Renaissance there are also frequent references to chaos as a lack of differentiation, a gaping void, a confused mass, an "undigested lump" (as George Sandys calls it in his translation of Ovid's Metamorphosis)." Sir Thomas Elyot warns in The Governor that if we "take awaie Order frome all thinges, what shulde than remaine? Certes nothing finally, except some man wold imagine eftesoones, Chaos, whiche of some is expounded, a confuse mixture." "The Prymer, or boke of Private Prayer (I 560) perhaps says it best: "That old confusion, which we call chaos, wherein without order, without fashion, confusedly lay the discordant seeds of things." "I

After the Renaissance, the classical suggestion that Chaos is the most ancient of all gods, the companion of Eros, and the stuff from which the world was made gradually grew obscure, and it finally dropped out of sight during the late eighteenth century. Enough of the classical resonances cling to Chaos for Pope to posit it as a deity (although of stunningly undistinguished lineage) in The Dunciad, where Dullness is described as "Daughter of Chaos and eternal

"George Sandys, Ovid's Metamorphosis, Englished, Mythologized, and Represented in Figures, ed. Karl K. Hulley and Stanley Vandersall (Lincoln: University of Nebraska Press, 1970), bk. I, p. 25.

"'Sir Thomas Elyot, The boke named The gouvenour, ed. Henry Herbert Stephan Croft (London: C. K. Paul, I 880), p. 3.

"Cited in the Oxford English Dictionary, ed. C. T. Onions et al. (Oxford: Clarendon, 1933), vol. 3, under "Chaos." The date given by the Short-Title Catalogue for this edition is 1560 , not 1559 , as the $O E D$ has it. 
Night" (I.I2). But the shift in tone is significant, and few references to Chaos as a god persist beyond that.

Slowly taking the place of this view of chaos was one that envisioned it as the antagonist to order. By the early nineteenth century, this view was predominant. Consider Edgar Allan Poe's tale "The Fall of the House of Usher" (1839). The preternatural and incestuously erotic energy that animates Madeline as she struggles to emerge from her coffin is in one sense an organizing force, for it brings her back to a living state; but these very exertions ensure that her approaching dissolution will be final, precipitating Roderick's death as well as hers. Madeline's exertions have their analogue in the storm that seems to animate the miasmic fog around the house. Just as Madeline's energy leads to the death of her twin, so the storm's expenditure of energy is linked with the disintegration of the house and the end of the family it sheltered and signified. These correlations imply that order and chaos are bound together in a dialectic. The more energy expended, the more certain the collapse into fragmentation and chaos. In the isolated and incestuously closed system formed by the House of Usher, Poe anticipated the second law of thermodynamics, which was being formulated by Sadi Carnot and others about this time. ${ }^{\prime 2}$

The popularization of thermodynamics during the 1860 s and I 870 os reinforced the antagonistic connection between order and chaos through predictions of a cosmic dissipation that would end with all heat sources everywhere being exhausted, resulting in the so-called "heat death" of the universe. Countering this pessimistic scenario was the awareness that in the short run (that is, in the eons while life still continued on earth), the release of thermal energy could run trains, fuel steamships, generate electricity. The power of heat engines was of a different kind as well as a different order from the mechanical advantage bestowed by pulleys, levers, and winches.

\footnotetext{
'-Sadi Carnot came close to formulating the second law in his 1824 treatise, Réflexions sur la puissance motrice $d u$ feu et sur les machines propres à développer cette puissance [Reflections on the motive power of fire and on the proper machine for developing this power]. In 1849 Lord Kelvin (William Thomson) wrote a paper that jointly attributed the second law to Carnot and to Rudolf Clausius, who pointed out the error that kept Carnot from arriving at a fully consistent formulation of the second law.
} 


\section{Chaos Bound}

Whereas mechanical energy was orderly, conveyed along visible lines of force, thermal energy was chaotic, driven by the fiery turbulence of the furnace and deriving its power from the random motions of molecules whizzing at unbelievable speeds. In "Turner Translates Carnot," Michel Serres argues that this vision of a turbulent, chaotic, immensely powerful energy inspired many of Turner's paintings (I982a, 54-64).

The ambiguity that once inhered in the concept of chaos was thus reconceptualized during the nineteenth century as a tension between a short-term release of energy and a long-term price paid for that release. Popularizations of this formula often took considerable liberties with interpretations of "long-term" and "short-term". Take Henry Adams. In The Education of Henry Adams, substantially completed in 1907 , Adams intimates that as man captures more and more powerful energies, history accelerates accordingly, until human intelligence (and implicitly the world) flies apart because it can no longer stand the strain. Yet in "A Letter to American Teachers of History," completed only three years later, he argues that human intellect has become progressively degraded since the Middle Ages, and that this degradation is inevitable because humans tap more and more powerful energies to keep them going. We see that the formula could be appropriated and used in very different ways, even by the same author.

Starting after World War I, and increasingly after World War II, the energy/dissipation ambiguity within chaos was shadowed by a corresponding ambiguity within order. On the one hand, order connoted stability, regularity, predictability. On the other, it signified a directive or a symbolic configuration one is not free to disobey, as in a military order or Foucault's "order of things" (1970). As chaos came to be seen as a liberating force, order became correspondingly inimical, associated with the mindless replication of military logic or with the oppressive control of a totalitarian state (or state of mind).

By 1960 , the stage was set for chaos to undergo a radical reevaluation. However, the multiple meanings it had acquired through centuries of commentary made it capable of diverse significations, even when it was cast in a generally positive light. This polysemy is evident in the differences between literary and scientific valuations of chaos. Literary theorists value chaos primarily because they are 
preoccupied with exposing the ideological underpinnings of traditional ideas of order. They like chaos because they see it as opposed to order. Chaos theorists, by contrast, value chaos as the engine that drives a system toward a more complex kind of order. They like chaos because it makes order possible.

The examples illustrate how the cultural field can energize different kinds of reevaluations, because the arrows in the field do not all point in the same direction. They also suggest that there is an interaction between the culture and the new paradigms, rather than a one-way flow of influence from one to the other. Even as the new paradigms bring into focus classical texts that may not have fitted very well into older traditions, these texts help to give traditional authority to the new paradigms. The idea that chaos could give rise to order, for example, is not without precedent. Henry Adams played with it; Poe ambiguously inscribed it; Hesiod gave it mythic authority. If these resonances had not existed, I think it unlikely that chaos theory would have aroused the widespread interest that it has. The entirely new concept is more likely to pass unheralded and unrecognized than one whose avatars echo through time. ${ }^{13}$

I suggested earlier that creative writing tends to carry along more of the cultural substrata than does writing within disciplines. Many postmodern texts self-reflexively play with their conditions of possibility, flaunting the stratification that in any case they are unlikely to escape. Consider Italo Calvino's Cosmicomics (1968). The stories in this collection are narrated by Qfwfq, who speaks with a human voice and consciousness and yet was present when the universe was not. The implication is that Qfwfq has evolved along with the universe, eventually becoming a being like us, and so able to communicate in a language we can understand. But the language that he (it?) uses is radically at odds with his attempt to tell us what it was like, for language is a relatively recent invention in the universe's history. Hence Qfwfq is constantly forced to qualify his descriptions, using words to describe what existed before language itself was born. Within this wildly anachronistic project, traces of ancient cos-

${ }^{13 .}$ This point is made in several of the essays in Borges, 1964 , including "Pascal's Sphere," "Kafka and His Precursors," "Avatars of the Tortoise," and "The Enigma of Edward FitzGerald." 
mogonic myths coexist with ideas taken from the new scientific paradigms.

In "The Form of Space," the narrator (presumably Qfwfq, although he is not identified) falls in a straight line through space, parallel to the fall of Ursula H'x, whom he desires, and Lieutenant Fenimore, whom he fears and detests as a potential rival. Throughout his fall, so reminiscent of the movement of Democritus's atoms, the narrator dreams of a swerve or clinamen that would bring him into contact with Ursula. Following the reasoning of Lucretius in $D e$ Rerum Natura, the narrator thinks of the clinamen as a longed-for release from the deadly order of the same. ${ }^{14}$

Significantly, he finds release not in actuality but in the curved lines of the letters constituting the text that writes him. He understands that if the lines of this writing were parallel, like his fall and those of his companions, signification would be impossible and hence nothing would happen in the story. Indeed, there could be no story for anything to happen in-no Ursula, no lieutenant, no narrator. The curvature of writing is thus equated with the swerve that Serres identifies with chaotic turbulence. Writing is turbulence, or more precisely, brings turbulence into being. The association suggests that before the world could exist, there had to be chaos; and simultaneously with chaos came writing, in the swerving inscriptions of the atoms when they first deviated from their linear paths. From this point of view, anachronism is not so much a fallacy as an inevitability. The story illustrates how contemporary ideas of chaos and signification can merge with ancient beliefs to form a narrative almost as stratified as culture itself.

It is this kind of play between new ideas and traditional formations that we are in danger of missing if we think of the present epistemic shift as homogeneous or total. The new paradigms break with old presuppositions; but language, tradition, and culture also affect our understanding of what is new in them. Working sometimes with, sometimes against the break with the past are the dynamics peculiar to a given site-the recursive techniques of non-

"Compare "Lucretius: Science and Religion," in Serres, 1982a:98-124. Whether Calvino's inspiration came directly from Lucretius or was mediated through Serres, I do not know. 
linear dynamics, the narrative conventions that a novelist such as Doris Lessing inherited from such predecessors as Conrad, James, Woolf, and Joyce, the anxiety of influence among critics when Derrida appeared on the scene. The resulting flow is turbulent rather than laminar, circling around in eddies and backwaters, responding to disturbances that affect the macroscopic qualities of the stream as it splits into tributary streams or converges into a swelling flood. For this reason my own narrative is nonlinear as it moves back and forth among scientific models, critical theory, and literary texts, following now one stream and now another, but always edging toward the alluvial plain where the currents run together to create the phenomena we call postmodernisms.

Subsequent chapters, reflecting the division between the two branches of chaos theory, are divided between texts that see chaos as a void from which something can emerge and those that see chaos as a complex configuration within which order is implicitly encoded. The first grouping, headed "Something out of Nothing," begins with a chapter that traces the transvaluation of entropy as it moves from classical thermodynamics to information theory. It shows that a crucial step in the reevaluation of chaos was the interpretation of randomness as maximum information. Chapter 3, on The Education of Henry Adams, shows similar ideas at work in a literary text. In The Education, the chaotic void becomes the space of creation. Through a combination of historical theorizing, personal trauma, and narrative strategies, The Education transforms its voids and ruptures into gaps from which radiates an energy that radically reorganizes whatever comes in contact with it. This reorganization is by no means unequivocally good; but it is finally irresistible, and therefore irrevocably bound up with human history.

Chapter 4 discusses the work of Prigogine and Stengers, focusing on their claim that the new paradigm reconciles being with becoming. This chapter, in addition to laying out the scientific basis for the order-out-of-chaos concept in irreversible thermodynamics and cosmology, speculates about the role of vision in shaping and directing scientific research programs. The "Something out of Nothing" section concludes with Chapter 5, on Stanislaw Lem. Like Adams, Lem conceives of chaos as a void from which something emerges. However, he works out a unique explanation for the creative power of 
chaos, seeing it as bound together with order in a complex dialectic through which chaos and order come to interpenetrate each other without losing their distinctive identities. In this respect Lem is poised on the threshold between the old and new paradigms, not going so far as to redefine chaos but believing that it must somehow be incorporated into our picture of the world. His work illustrates that transitions to new paradigms are never homogeneous or uniquely determined. Even when many of the same elements are present, they can be combined differently by writers with different backgrounds and agendas.

The second group of chapters, headed "The Figure in the Carpet," begins with a discussion of the strange-attractor branch of chaos theory. Among the theories discussed in chapter 6 are Feigenbaum's universality theory, Mandelbrot's fractal geometry, Robert Shaw's interpretations of chaos as information, and Kenneth Wilson's quantum field theory. The chapter concludes with a discussion of James Gleick's Chaos, showing how its narrative patterns conflict with some of the new paradigm's fundamental assumptions. Chapter 7 discusses poststructuralist theories, focusing on the work of Jacques Derrida, Roland Barthes, and Michel Serres. It experiments with creating a model that can account for divergences as well as isomorphisms between fields. It suggests that analogies are best understood through an ecology of ideas, and that differences can be accounted for through various disciplinary economies and the equivocations that result when a concept is imported from one discipline to another.

Chapter 8 addresses the politics of chaos. Among the ideological issues raised by the new paradigms are the valorization of local knowledge and the corresponding repudiation of global theories. But the situation is more complex than it may at first appear. Chaos theory, although it recognizes the importance of scale, does so in order to globalize more effectively. Similarly, poststructuralism finds a new globalizing imperative in its insistence that there can be no global theories. These complexities suggest that ideological stances cannot be fully understood apart from the disciplinary contexts in which they are embedded.

Chapter 9 deals with Doris Lessing's Golden Notebook (1962). The politics of chaos are central to the text's narrative organization, 
as well as to its wide-ranging analyses of Marxism, sexism, and racism. The Golden Notebook has many of the characteristics associated with the new paradigms - a problematic relation between local sites and global theories, an interest in recursive symmetries as a principle of organization, an awareness of how small fluctuations can effect large-scale changes. These analogies cannot be explained by scientific influence, for Lessing knew little or nothing of chaos theory. Rather, they represent the independent re-creation by Lessing of a chaos theory of her own. Convinced that society is on the verge of cracking up, Lessing's narrator desperately tries to find clues to a new synthesis in her own disintegrating psyche. This text thus provides insight into the broad cultural movements that underlie the new paradigms.

The two major sections, "Something out of Nothing" and "The Figure in the Carpet," are framed by the present introductory chapter and a concluding chapter that locates chaos theory within the narrative of postmodernism. Chapter io argues that postmodernism can be understood as a continuing process of denaturing, that is, of realizing that concepts once considered natural are in fact social constructions. The denatured concepts include language, time, context, and, increasingly as postmodernism progresses, the human. Chaos has its frightening as well as its liberating aspects. Fragmentation and unpredictability are not, I argue, always cause for celebration.

The title of this study, Chaos Bound, was chosen because it hints at the complexities of these contemporary inscriptions of chaos. One cluster of meanings centers on the sense of "bound" as a limit line or boundary. Bringing into view a third territory that lies between order and disorder, chaos theory draws boundaries where previously there was only bifurcation. It also emphasizes the importance of boundaries within systems.

A second set of meanings comes into play with the sense of "bound" as confinement or bondage. In The Postmodern Condition (1984) Lyotard sees some aspects of chaos models, particularly fractal geometry, as promising a release from totalizing narratives. Yet other aspects of chaos theory bring this vision into question, for they embody a shift in perspective away from the individual and toward systemic organization. As we move into an episteme that sees the human as a social construct, it is an open question whether individu- 


\section{Chaos Bound}

ality, in its traditional Western guises, can continue to be seen as a liberating or energizing force.

A third cluster of meanings emerges from the sense of "bound" as resolve and determination, especially to attain a destination. The emergence of the new scientific paradigms are authorized by the same cultural developments that have led to literary and critical postmodernisms. At once promising an escape from oppressive order and implying that even chaos can be orderly, these theories replicate the ambiguity at the heart of postmodern culture. Whether arrival at this destination will transform society, as it has already transformed some scientific and literary sites, is a question beyond the bounds of Chaos Bound. Nevertheless, it seems clear that local fluctuations are in the process of being magnified into large-scale changes. Chaos both exemplifies this process and names it. 